\title{
Effect of artificial shrinkage on clinical outcome in fresh blastocyst transfer cycles
}

\author{
Yong Soo Hur ${ }^{1,2}$, Jeong Hyun Park² , Eun Kyung Ryu' ', Hae Jin Yoon' ', San Hyun Yoon ${ }^{3}$, Chang Young Hur', Won Don Lee', \\ Jin Ho Lim ${ }^{1}$ \\ ${ }^{1}$ Maria Fertility Hospital, Seoul; ${ }^{2}$ Department of Anatomy, Kangwon National University School of Medicine, Chuncheon; ${ }^{3}$ Fertility Research Center, \\ Seoul, Korea
}

Objective: This study aimed to determine the safety and clinical effect of artificial shrinkage (AS) in terms of assisted hatching of fresh blastocysts. Also, we evaluated the correlation between patient age and the effect of AS on clinical outcome.

Methods: Two AS methods, using a 29-gauge needle and laser pulse, were compared. Seventy-three blastocysts were shrunk using a 29-gauge needle and the same number of other blastocysts were shrunk by a laser pulse. We evaluated the shrunken blastocysts hourly and considered them viable if they re-expanded $>70 \%$. Blastocyst transfer cycles $(n=134)$ were divided into two groups: a control group consisted of the cycles whose intact embryos were transferred $(n=100)$, while the AS group consisted of the cycles whose embryos were replaced following AS $(\mathrm{n}=34)$. The implantation and pregnancy rates of the control group and AS group were compared $(p<0.05)$.

Results: The re-expansion rates of the 29-gauge needle and laser pulse AS groups were similar (56 [76.7\%] vs. 62 [84.9\%], respectively). All of the remaining shrunken blastocysts were re-expanded within 2 hours. There was no degeneration of shrunken blastocysts. The total and clinical pregnancy rate of the AS group (23 [67.6\%]; 20 [58.8\%], respectively) was significantly higher than that of the control group (47 [47.0\%]; 39 [39.0\%], respectively). In the older patient group, there was no difference in the clinical outcomes between the AS and control groups.

Conclusion: These results suggest that AS of blastocoele cavity, followed by the transfer, would be a useful approach to improve the clinical outcome in cycles in which fresh blastocyst stage embryos are transferred.

Keywords: Artificial Shrinkage; Embryo Transfer; Assisted Hatching; In Vitro Fertilization; Human

\section{Introduction}

In developing from the morula to the blastocyst, embryos undergo dramatic morphologic changes. When blastocysts expand, fluid gradually accumulates in the blastocoel-mediated sodium pump ( $\mathrm{Na}^{+}$, $\mathrm{K}^{+}$, ATPase) [1], resulting in increased pressure on both the trophec-

Received: Apr 18, 2011 - Revised: Jun 1, 2011 · Accepted: Jun 8, 2011 Corresponding author: Yong Soo Hur

Maria Fertility Hospital, 103-11 Sinseol-dong, Dondaemun-gu, Seoul 130-812, Korea

Tel: +82-2-2250-5572 Fax:+82-2-2250-5585 E-mail:geaher@mariababy.com

*Presented at 20th World Congress of Fertility and Sterility, Munich, Germany.

This is an Open Access article distributed under the terms of the Creative Commons Attribution Non-Commercial License (http://creativecommons.org/licenses/by-nc/3.0/) which permits unrestricted non-commercial use, distribution, and reproduction in any medium, provided the original work is properly cited. toderm and zona pellucida (ZP). At the same time, trophectoderm cells secrete lysins that are involved in ZP thinning and hatching. Expansion and ZP thinning occur in mammalian blastocysts prior to hatching [2-4]. Contraction-expansion cycles, as well as expansion and ZP thinning of blastocysts, have been documented by time-lapse video recording $[5,6]$.

The rationale of artificial shrinkage (AS) is based on contraction-expansion cycles and cytoplasmic extension of the trophectoderm of the blastocyst. The mechanism of blastocoel collapse and recovery of trophectoderm rupture is unclear. Whatever causes a collapse of the blastocyst, shrunken blastocysts have the potential to gradually recover their spheroidal shape. The AS technique is widely used and considered an essential step to improve the efficiency of vitrification. Researchers using AS before vitrification of blastocysts have reported 
higher implantation and clinical pregnancy rates [7-12].

The advantage of AS is that ice crystal formation can be avoided by reducing the fluid content of the blastocoel [7]. Some researchers assume that creating a large hole in the ZP with any of various AS tools (i.e., glass micro-needle, injection needle, micropipetting with a handdrawn Pasteur pipette, 29-gauge needle, or laser pulse) has some kind of effect on assisted hatching $(\mathrm{AH})[13,14]$. In the process of AS, various AS tools create physical damage to the ZP as well as to the trophectoderm. It is assumed that re-expanding the blastocyst during warming eases hatching through the ZP-damaged areas. In fact, most thawed blastocysts with AS have a higher hatching rate, whether or not they hatch through the ZP-damaged areas.

Although blastocyst transfer has provided good clinical results, researchers have become increasingly aware that it is more difficult to obtain a certain pregnancy rate $[15,16]$. Thus, some researchers have attempted to include an additional process before blastocyst transfer to achieve a higher pregnancy rate. Fong et al. [17] attempted to remove the total ZP with pronase before blastocyst transfer, Turker [18] attempted to make a large hole in the ZP with acid Tyrode's solution, and Goto et al. [19] attempted to stimulate the endometrium by injection of embryo culture supernatant into the uterus. We attempted to use the AS technique on fresh blastocysts before transfer.

The objective of this study was to determine the impact of AS on fresh blastocysts, and to determine whether or not AS had an effect, such as assisted hatching, by comparing clinical outcomes. We evaluated the correlation between patient age and the effect of AS on clinical outcome.

\section{Methods}

\section{Patients and IVF}

Patients who entered our blastocyst transfer program and agreed to perform assisted hatching were $\geq 36$ years of age or had previous repeat failures of cleavage-stage embryo transfers. Between July and December 2008, 100 cycles were randomly selected as a control group. We applied the 29-gauge needle AS technique to 34 cycles. In each group (control and AS groups), we evaluated the effect of the AS technique on patients $\geq 36$ years of age. Patients were treated with GnRH agonist and hMG in according to a long- or a short-treatment protocol. When two and more follicles reached $18 \mathrm{~mm}$ in diameter, 5,000 IU of hCG (Ovidrel; Merk-Serono, Bari, Italy) was administered. Oocytes were retrieved transvaginally 36-38 hours after hCG injection and the oocytes were inseminated by conventional IVF or ICSI.

\section{Embryo culture}

Fertilization was assessed 15-18 hours after insemination by the presence of two pronuclei. Zygotes were washed and cultured in groups < 5 in Sydney IVF Cleavage Medium (CM; COOK, Brisbane, Australia) for 48 hours, then in Sydney IVF Blastocyst Medium (BM; COOK) for another 48-71 hours. All culturing of embryos was performed in a $\mathrm{CO}_{2}: \mathrm{O}_{2}: \mathrm{N}_{2}$ (6\%:5\%:89\%) environment. On day 5, blastocysts were scored depending on the developmental stage and graded according to quality criteria [20].

\section{Artificial shrinkage of expanded blastocysts}

Son et al. [8] previously described the mechanical technique of shrinkage using two 29-gauge needles. Briefly, after holding the expanded blastocyst with the flat side of a needle and placing the inner cell mass (ICM) at the 12 or 6 o'clock position, a needle was pushed through the trophectoderm cell in the blastocoel cavity until the cavity shrank. Contraction of the blastocysts was observed after 30 seconds to 1 minute. After complete shrinkage of the blastocoel, the blastocysts were cultured in BM before embryo transfer.

A laser system (ZILOS-tk Laser Zona Drill System; Hamilton Thorn Bioscience, Inc., Beverly, MA, USA) was introduced to compare with the 29-gauge needle AS. The process of the AS was followed by Mukaida's method [14]. The ICM should be placed away from the targeted point of the laser pulse. A single laser pulse ( $300 \mu \mathrm{s}$ ) was delivered to the outer periphery of the trophectoderm at the junction between the cells. It was sufficient to induce complete shrinkage within 1-2 minutes. After complete shrinkage of the blastocoel, the blastocysts were cultured in the same media.

\section{Evaluation of impact of AS on fresh blastocysts}

Several previous studies have concluded that AS before vitrification is a useful approach for improving clinical outcomes and guaranteeing the safety of the AS technique for human blastocysts. With a slightly different perspective, we attempted to determine the impact of AS on fresh blastocysts that were surplus embryos for cryopreservation. A 29-gauge needle and laser pulse AS were compared. Seventythree blastocysts were shrunk by using a 29-gauge needle and the same number of other blastocysts were shrunk using a laser pulse. After AS was performed, every blastocyst was evaluated hourly until the re-expansion rate was up to $70 \%$. We confirmed that $70 \%$ of the re-expanded blastocysts were viable. After observing for 2 hours, the highest quality blastocysts were cryopreserved and the others were discarded.

Assisted hatching of blastocysts and AS before vitrification of every blastocyst has already been permitted by the policy of the Maria Fertility Hospital Institutional Review Board. The evaluation of re-expanded blastocysts was treated as a part of the whole freezing process.

\section{Blastocyst transfer and assessment of pregnancy}

On the morning of embryo transfer (ET), we selected 2 or 3 good 
quality blastocysts and performed AS before transfer. After 2 or 2.5 hours, we selected the best quality re-expanded blastocysts and transferred them. We were limited to transfering two blastocysts. Only a few patients received three blastocysts, based on their clinical history (i.e., repeat failures or age $\geq 36$ years). Pregnancy was assessed by serum hCG 14 days after administration of progesterone, then implantation was confirmed by the presence of a gestational sac. Clinical pregnancy was confirmed by the presence of fetal heart activity.

\section{Statistics}

The data were examined by $x^{2}$ analysis to determine whether differences in implantation and pregnancy rates were significant between the control and AS groups.

Table 1. The time required for re-expansion ( $\geq 70 \%$ ) of shrunk blastocysts

\begin{tabular}{lcccc} 
& No. of & \multicolumn{2}{c}{ Time for re-expansion (hr) } & \multirow{2}{*}{$\begin{array}{c}\text { Survival } \\
\text { rate }\end{array}$} \\
\cline { 3 - 4 } & blastocysts & 1 & 2 & 2 \\
\hline 29-gauge needle AS & 73 & $56(76.7)$ & $17(23.3)$ & $73(100)$ \\
Laser pulse AS & 73 & $62(84.9)$ & $11(15.1)$ & $73(100)$ \\
Total & 146 & $118(80.8)$ & $28(19.2)$ & $146(100)$ \\
\hline
\end{tabular}

Values are presented as number (\%).

AS, artificial shrinkage.

\section{Results}

We evaluated the impact of AS on fresh blastocysts by observing the rate of re-expansion to $70 \%$ of the original volume. The 29 -gauge needle and laser pulse AS were compared (Table 1). Seventy-three blastocysts were shrunk by using a 29-gauge needle; 56 blastocysts (76.7\%) were re-expanded up to at least 70\% within 1 hour (Figure 1). The remaining blastocysts (17 [23.3\%]) were re-expanded within 2 hours.

The same number of other blastocysts were shrunk using a laser pulse; 62 blastocysts (84.9\%) were re-expanded up to at least $70 \%$ within 1 hour. The remaining blastocysts (11 [15.1\%]) were re-expanded within 2 hours. All blastocysts were re-expanded up to $70 \%$ within 2 hours. There was no degeneration of the shrunken blastocysts.

The re-expansion rates using the 29-gauge needle and laser pulse AS were similar. Nearly $80 \%$ of the shrunken blastocysts were re-expanded within 1 hour. All of the remaining shrunken blastocysts were re-expanded within 2 hours.

Based on previous results, we applied the AS technique clinically. Table 2 summarizes the clinical outcome comparing the control and AS groups. Laser pulse AS had not been applied by that time. The mean number of blastocyst transfers was quite similar between the control and AS groups. The implantation rate of the 29-gauge needle
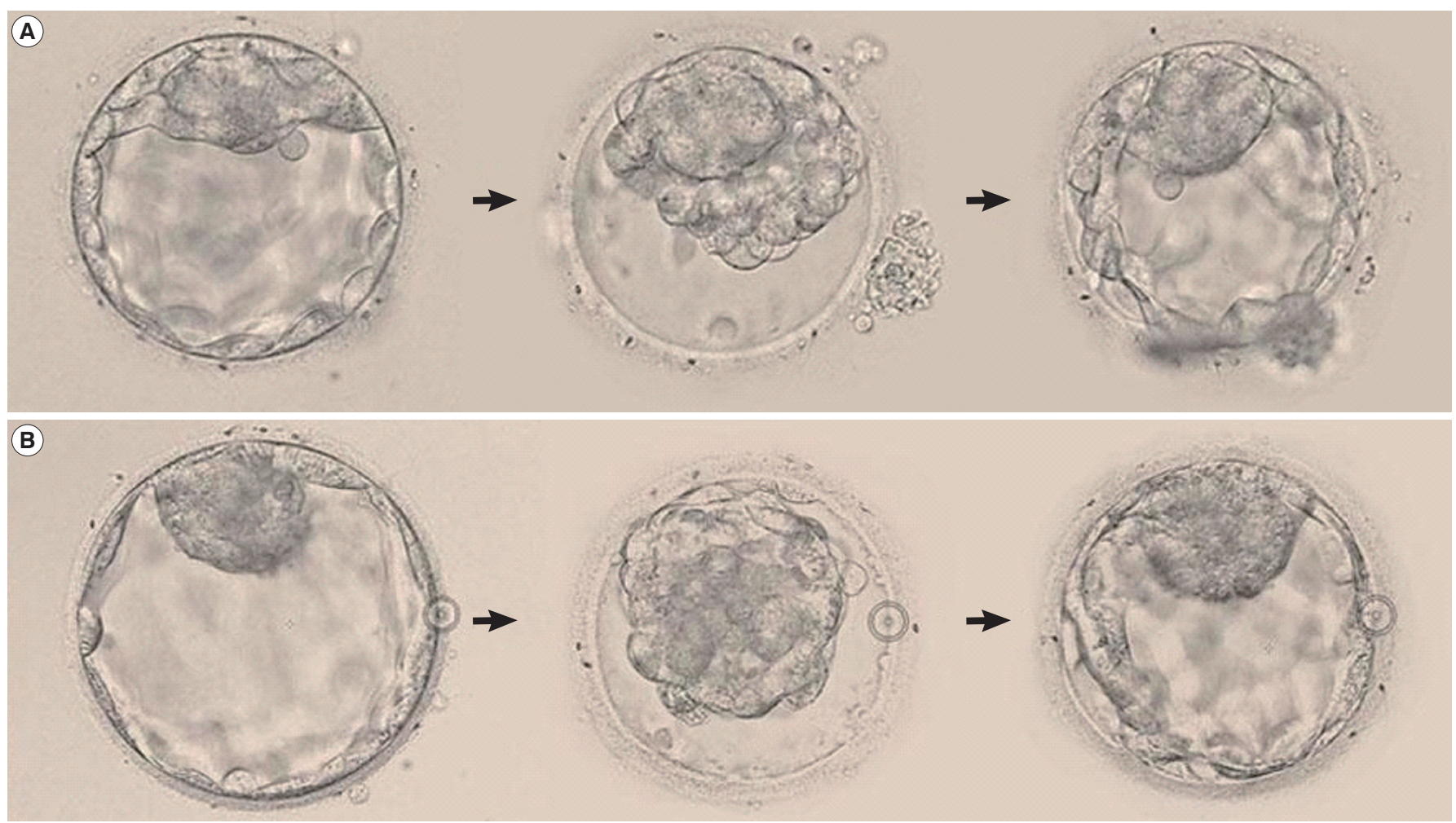

Figure 1. Blastocyst shrinkage and re-expansion ( $\geq 70 \%$ ) using a 29-gauge needle (A) and laser pulse (B) (magnification $\times 200)$. 
Table 2. The clinical outcomes between the control and artificial shrinkage (AS) groups

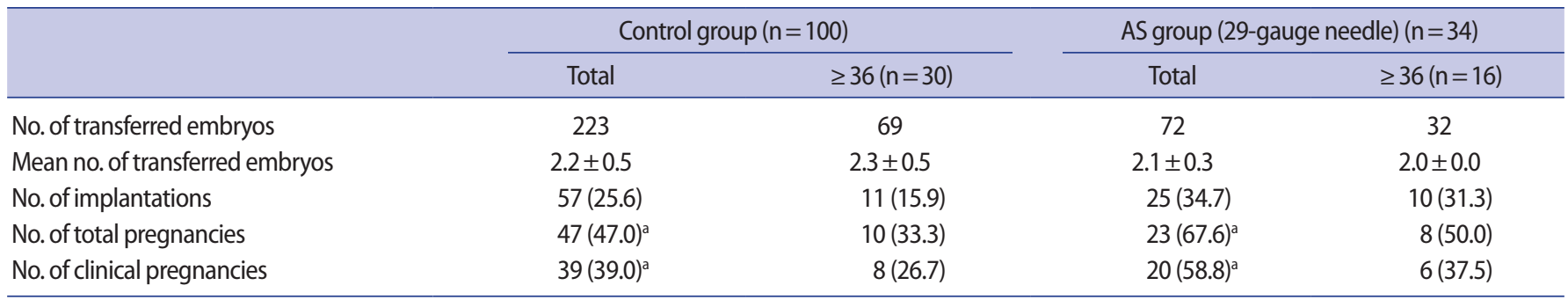

Values are presented as mean \pm SD or number (\%).

${ }^{a} p<0.05$; Control: Non-AS group.

AS group was higher than the control group, but the difference was not statistically significant. The total and clinical pregnancy rates of the AS group were significantly higher than those of the control group. In patients with an age of $\geq 36$ years, the clinical outcome of the AS group was higher than that of the control group, but the difference was not statistically significant.

\section{Discussion}

This study demonstrated that AS of fresh blastocysts before transfer had very encouraging clinical results. We confirmed the safety of laser pulse AS as well as 29-gauge needle AS on fresh blastocysts. Our laboratory has previously reported a vitrification method for human blastocysts on electron microscopy grids combined with AS using a 29-gauge needle [8], but we have also published serial studies which led to the previous study [21-24]. Thus, we have considerable experience with AS using a fine needle. Due to our accumulated experience, we reasoned that physical damage would be minimized.

As mentioned earlier, AS before vitrification of blastocysts and $\mathrm{AH}$ of blastocysts has already been permitted by the policy of the Maria Fertility Hospital Institutional Review Board. The evaluation of re-expanded blastocysts was treated as a part of the whole freezing process. Most of the blastocysts designated for vitrification were re-shrunk in the media for cryopreservation. Some blastocysts that did not shrink in the freezing media were cryopreserved without going through AS. We had experienced that sometimes a blastocyst was not shrink completely. We could not find the reason why some blastocysts shrink incompletely.

During embryo biopsy for pre-implantation genetic diagnosis (PGD), a large hole is created in the ZP of the blastocyst. However, the safety of biopsy for PGD is still a matter of debate [25-27]. It appears that the effect of AS is less extensive than PGD by comparing several clinical outcomes. We recommend that additional study is needed at the DNA/cellular level to determine the effects of AS on fresh blastocysts.

The advantage of AS is improving blastocyst viability after vitrification, which prevents ice crystal damage by reducing fluid within the blastocoel cavity [7]. Other researchers have estimated that there may be an effect of assisted hatching caused by the formation of a large hole in the ZP, especially with 29-gauge needle AS [8]. We have confidence that there is a helpful effect of AS in terms of $\mathrm{AH}$. Most previous reports on the effect of $\mathrm{AH}$ have performed $\mathrm{AH}$ on the cleavage stage using acid Tyrode's solution, a mechanical method, or a laser $[28,29]$. Enzymatic treatment of the ZP to completely remove the ZP before blastocyst transfer has resulted in a high implantation rate [30]. AH at the blastocyst stage needs a more careful approach. Some researchers claim that there is no need to do $\mathrm{AH}$ on blastocysts because they have an intrinsically higher viability than cleavage-stage embryos [31]. Our study showed that the AS technique is not harmful to the survival rate of blastocysts, and is worthwhile as a new $\mathrm{AH}$ technique to perform on blastocysts before transfer.

Single embryo transfer (SET) is the effective method for reducing the IVF multiple pregnancy rate [32]. For SET to be successful, it is crucial to have confidence in embryo viability [33]. Blastocyst transfer is a better method for diminishing the controversy over embryo viability. Combining a single blastocyst and the AS technique before transfer would be a better approach to SET.

In this study, the tendency for AS to improve the rate of blasctocysts having successful implantation and pregnancy in patients aged $\geq 36$ years was insignificant. Further study is needed to determine whether or not AS is helpful in older patients.

In conclusion, this study showed that AS of human blastocysts before transfer is a clinically useful method.

\section{Conflict of interest}

No potential conflict of interest relevant to this article was reported.

\section{References}

1. Houghton FD, Humpherson PG, Hawkhead JA, Hall CJ, Leese HJ. $\mathrm{Na}^{+}, \mathrm{K}^{+}$, ATPase activity in the human and bovine preimplantation embryo. Dev Biol 2003;263:360-6. 
2. Biggers JD. Reflections on the culture of the preimplantation embryo. Int J Dev Biol 1998;42:879-84.

3. Watson AJ, Barcroft LC. Regulation of blastocyst formation. Front Biosci 2001;6:D708-30.

4. Veeck LL, Zaninovic N. An atlas of human blastocysts. New York: Parthenon Pub. Group; 2003.

5. Glass RH, Lin TP, Florence J. Mouse blastocyst re-expansion following puncture and treatment with inhibitors. J Reprod Fertil 1973;35:533-6.

6. Gonzales DS, Bavister BD. Zona pellucida escape by hamster blastocysts in vitro is delayed and morphologically different compared with zona escape in vivo. Biol Reprod 1995;52:470-80.

7. Vanderzwalmen P, Bertin G, Debauche C, Standaert V, van Roosendaal $E$, Vandervorst $M$, et al. Births after vitrification at morula and blastocyst stages: effect of artificial reduction of the blastocoelic cavity before vitrification. Hum Reprod 2002;17:744-51.

8. Son WY, Yoon SH, Yoon HJ, Lee SM, Lim JH. Pregnancy outcome following transfer of human blastocysts vitrified on electron microscopy grids after induced collapse of the blastocoele. Hum Reprod 2003;18:137-9.

9. Hiraoka K, Kinutani M, Kinutani K. Blastocoele collapse by micropipetting prior to vitrification gives excellent survival and pregnancy outcomes for human day 5 and 6 expanded blastocysts. Hum Reprod 2004;19:2884-8.

10. Mukaida T. Artificial shrinkage (AS) of blastocoele prior to cooling step of vitrification improves the survival rate of vitrified human blastocysts. Fertil Steril 2004;82:S115.

11. Mukaida T, Takahashi K, Goto T, Oka C. Ultra-rapid vitrification using a cryoloop technique for human blastocyst cryopreservation. Fertil Steril 2005;84:S476.

12. Chen SU, Lee TH, Lien YR, Tsai YY, Chang LJ, Yang YS. Microsuction of blastocoelic fluid before vitrification increased survival and pregnancy of mouse expanded blastocysts, but pretreatment with the cytoskeletal stabilizer did not increase blastocyst survival. Fertil Steril 2005;84 Suppl 2:1156-62.

13. Desai N, Szeptycki J, Scott M, Abdelhafez FF, Goldfarb J. Artificial collapse of blastocysts before vitrification: mechanical vs. laser technique and effect on survival, cell number, and cell death in early and expanded blastocysts. Cell Preserv Technol 2008;6:181-9.

14. Mukaida T, Oka C, Goto T, Takahashi K. Artificial shrinkage of blastocoeles using either a micro-needle or a laser pulse prior to the cooling steps of vitrification improves survival rate and pregnancy outcome of vitrified human blastocysts. Hum Reprod 2006;21: 3246-52.

15. Alves da Motta EL, Alegretti JR, Baracat EC, Olive D, Serafini PC. High implantation and pregnancy rates with transfer of human blastocysts developed in preimplantation stage one and blasto- cyst media. Fertil Steril 1998;70:659-63.

16. Schwärzler $P$, Zech $H$, Auer M, Pfau K, Göbel G, Vanderzwalmen $P$, et al. Pregnancy outcome after blastocyst transfer as compared to early cleavage stage embryo transfer. Hum Reprod 2004;19: 2097-102.

17. Fong CY, Bongso A, Ng SC, Kumar J, Trounson A, Ratnam S. Blastocyst transfer after enzymatic treatment of the zona pellucida: improving in-vitro fertilization and understanding implantation. Hum Reprod 1998;13:2926-32.

18. Turker M. Relevance of assisted hatching with blastocyst stage transfer. The Embryologists 2000;2:1-15.

19. Goto S, Kadowaki T, Hashimoto H, Kokeguchi S, Shiotani M. Stimulation of endometrium embryo transfer can improve implantation and pregnancy rates for patients undergoing assisted reproductive technology for the first time with a high-grade blastocyst. Fertil Steril 2009;92:1264-8.

20. Gardner DK, Lane M, Stevens J, Schlenker T, Schoolcraft WB. Blastocyst score affects implantation and pregnancy outcome: towards a single blastocyst transfer. Fertil Steril 2000;73:1155-8.

21. Cha JH, Kim HJ, Yoon HJ, Yoon SH, Kang CW, Lim JH. Vitrification of the blastocyst-stage embryos derived from TESE/PESA. Fertil Steril 2007;88:S358.

22. Kim DJ, Kim HJ, Yoon HJ, Yoon SH, Lee WD, Lim JH. Day 5 blastocyst has higher viability compared to day 6 blastocyst as vitrified: clinical outcome of 1074 cases. Fertil Steril 2008;90:S291.

23. Lee SY, Jae BS, Lee SY, Son WY, Yoon SH, Lim JH. Optimization of a thawing method for human expanded blastocysts vitrified using EM-Grid after artificial shrinkage. Fertil Steril 2003;80:147-8.

24. Lee SY, Son WY, Yoon SH, Lim JH. Clinical-pregnancy outcome after vitrification of blastocysts produced from in vitro maturation cycles. Fertil Steril 2007;88:1449-51.

25. Clouston HJ, Herbert M, Fenwick J, Murdoch AP, Wolstenholme J. Cytogenetic analysis of human blastocysts. Prenat Diagn 2002; 22:1143-52.

26. Blake DA, Forsberg AS, Johansson BR, Wikland M. Laser zona pellucida thinning--an alternative approach to assisted hatching. Hum Reprod 2001;16:1959-64.

27. Dokras A, Sargent IL, Ross C, Gardner RL, Barlow DH. Trophectoderm biopsy in human blastocysts. Hum Reprod 1990;5:821-5.

28. Kokkali G, Vrettou C, Traeger-Synodinos J, Jones GM, Cram DS, Stavrou D, et al. Birth of a healthy infant following trophectoderm biopsy from blastocysts for PGD of beta-thalassaemia major. Hum Reprod 2005;20:1855-9.

29. Lyu QF, Wu LQ, LiYP, Pan Q, Liu DE, Xia K, et al. An improved mechanical technique for assisted hatching. Hum Reprod 2005;20: 1619-23.

30. Fong $\mathrm{CY}$, Bongso A, Sathananthan $\mathrm{H}, \mathrm{Ho} \mathrm{J,} \mathrm{Ng} \mathrm{SC}$. Ultrastructural 
observations of enzymatically treated human blastocysts: zonafree blastocyst transfer and rescue of blastocysts with hatching difficulties. Hum Reprod 2001;16:540-6.

31. Gardner DK, Schoolcraft WB, Wagley L, Schlenker T, Stevens J, Hesla J. A prospective randomized trial of blastocyst culture and transfer in in-vitro fertilization. Hum Reprod 1998;13:3434-40.

32. Matorras R, Matorras F, Mendoza R, Rodríguez M, Remohí J, Rodríguez-Escudero FJ, et al. The implantation of every embryo facilitates the chances of the remaining embryos to implant in an
IVF programme: a mathematical model to predict pregnancy and multiple pregnancy rates. Hum Reprod 2005;20:2923-31.

33. van Montfoort AP, Fiddelers AA, Janssen JM, Derhaag JG, Dirksen $C D$, Dunselman GA, et al. In unselected patients, elective single embryo transfer prevents all multiples, but results in significantly lower pregnancy rates compared with double embryo transfer: a randomized controlled trial. Hum Reprod 2006;21: 338-43. 